\title{
Matter-Antimatter Coexistence Method for Finite Density QCD toward a Solution of the Sign Problem
}

\author{
Hideo Suganuma \\ Department of Physics, Graduate School of Science, Kyoto University, Kyoto, Japan \\ Email:suganuma@scphys.kyoto-u.ac.jp
}

How to cite this paper: Suganuma, $\mathrm{H}$. (2017) Matter-Antimatter Coexistence Method for Finite Density QCD toward a Solution of the Sign Problem. Journal of Modern Physics, 8, 2034-2041.

https://doi.org/10.4236/jmp.2017.812123

Received: October 27, 2017

Accepted: November 25, 2017

Published: November 28, 2017

Copyright $\odot 2017$ by author and Scientific Research Publishing Inc. This work is licensed under the Creative Commons Attribution International License (CC BY 4.0).

http://creativecommons.org/licenses/by/4.0/

\begin{abstract}
Toward the lattice QCD calculation at finite density, we propose "matter-antimatter coexistence method", where matter and anti-matter systems are prepared on two parallel $\mathbf{R}^{4}$-sheets in five-dimensional Euclidean space-time. We put a matter system $M$ with a chemical potential $\mu \in \mathbf{C}$ on a $\mathrm{R}^{4}$-sheet, and also put an anti-matter system $\bar{M}$ with $-\mu^{*}$ on the other $\mathbf{R}^{4}$-sheet shifted in the fifth direction. Between the gauge variables $U_{v} \equiv \mathrm{e}^{i a g A_{v}}$ in $M$ and $\tilde{U}_{v} \equiv \mathrm{e}^{i a g \tilde{A}_{v}}$ in $\bar{M}$, we introduce a correlation term $S_{\lambda} \equiv \sum_{x, v} 2 \lambda\left\{N_{c}-\operatorname{Re} \operatorname{tr}\left[U_{v}(x) \tilde{U}_{v}^{\dagger}(x)\right]\right\} \simeq \sum_{x} \frac{1}{2} \lambda a^{2}\left\{A_{\nu}^{a}(x)-\tilde{A}_{\nu}^{a}(x)\right\}^{2}$ with a real parameter $\lambda$. In one limit of $\lambda \rightarrow \infty$, a strong constraint $\tilde{U}_{v}(x)=U_{v}(x)$ is realized, and therefore the total fermionic determinant becomes real and non-negative, due to the cancellation of the phase factors in $M$ and $\bar{M}$, although this system resembles QCD with an isospin chemical potential. In another limit of $\lambda \rightarrow 0$, this system goes to two separated ordinary QCD systems with the chemical potential of $\mu$ and $-\mu^{*}$. For a given finite-volume lattice, if one takes an enough large value of $\lambda$, $\tilde{U}_{v}(x) \simeq U_{v}(x)$ is realized and phase cancellation approximately occurs between two fermionic determinants in $M$ and $\bar{M}$, which suppresses the sign problem and is expected to make the lattice calculation possible. For the obtained gauge configurations of the coexistence system, matter-side quantities are evaluated through their measurement only for the matter part $M$. The physical quantities in finite density QCD are expected to be estimated by the calculations with gradually decreasing $\lambda$ and the extrapolation to $\lambda=0$. We also consider more sophisticated improvement of this method using an irrelevant-type correlation.
\end{abstract}




\section{Keywords}

Lattice QCD, Finite Density, Sign Problem, QCD Phase Diagram

\section{Introduction}

Nowadays, quantum chromodynamics (QCD) has been established as the fundamental theory of strong interaction. Together with the success of perturbative QCD for high-energy processes of hadron reactions, the lattice QCD Monte Carlo simulation has been a powerful tool to analyze nonperturbative aspects of QCD, after the formulation of lattice QCD and its numerical success [1]. Indeed, lots of studies of the QCD vacuum, hadrons and the quark-gluon plasma have been done for both zero-temperature and finite-temperature in lattice QCD.

Finite density QCD is also important to understand the QCD diagram, nuclear systems and neutron stars, and it is desired to perform the lattice QCD analysis as the first-principle calculation of the strong interaction. However, there appears a serious problem called the "sign problem" [2] [3] in the practical lattice QCD calculation at finite density. This problem originates from the complex value including minus sign of the QCD action and the fermionic determinant at finite density even in the Euclidean metric [4].

At finite density with the chemical potential $\mu$, the Euclidean QCD action $S[A, \psi, \bar{\psi} ; \mu]$ is generally complex,

$$
S[A, \psi, \bar{\psi} ; \mu]=S_{G}[A]+\int \mathrm{d}^{4} x\left\{\bar{\psi}\left(D+m+\mu \gamma_{4}\right) \psi\right\} \in \mathbf{C},
$$

with the gauge action $S_{G}[A] \in \mathbf{R}$ and covariant derivative $D^{v} \equiv \partial^{v}+i g A^{v}$. (In this paper, we use hermite $\gamma$-matrices of $\gamma_{\mu}^{\dagger}=\gamma_{\mu}$ in the Euclidean metric.) Therefore, one cannot identify the action factor as a probability density in the QCD generating functional, unlike ordinary lattice QCD calculations. Also, the fermionic determinant at finite density generally takes a complex value [4], and its phase factor is drastically changed depending on the gauge configuration in a large-volume lattice, so that the Monte Carlo importance sampling loses its efficiency, which makes the numerical analysis highly difficult for the evaluation of the integral. This is the sign problem.

The sign problem has been a longstanding difficult problem in computational physics, and widely appears in various region of statistical physics, condensed matter physics and QCD physics at finite density. Then, it is desired to develop some methods to suppress the sign problem in many fields in theoretical physics.

In this paper, we propose a new method of "matter-antimatter coexistence method" [5] utilizing the charge conjugation symmetry for the practical lattice QCD calculation at finite density, aiming at a possible solution of the sign problem. The purpose of this method is to suppress the sign problem and then to enlarge the calculable region of the QCD phase diagram at finite density and temperature. The organization of this paper is as follows. In Section 2, we 
propose a new theoretical method of "matter-antimatter coexistence method" in Euclidean QCD at finite density, and show its actual procedure for the lattice calculation. Section 3 will be devoted to the summary and the conclusion.

\section{Matter-Antimatter Coexistence Method}

In this section, we introduce the matter-antimatter coexistence method for general complex chemical potential $\mu \in \mathbf{C}$. In this method, we use phase cancellation of the fermionic determinants between a matter system with $\mu$ and an anti-matter system with $-\mu^{*}$, which generally holds in QCD at finite density.

\subsection{General Property of QCD at Finite Density}

To begin with, we start from the general property of finite-density QCD [4],

$$
S[A, \psi, \bar{\psi} ; \mu]^{*}=S\left[A, \psi, \bar{\psi} ;-\mu^{*}\right],
$$

for the Euclidean QCD action $S[A, \psi, \bar{\psi} ; \mu]$ in the presence of the chemical potential $\mu \in \mathbf{C}$.

For instance, in continuum QCD, one finds

$$
\left[\bar{\psi}\left(D+m+\mu \gamma_{4}\right) \psi\right]^{*}=\bar{\psi}\left(D+m-\mu^{*} \gamma_{4}\right) \psi,
$$

which leads to Equation (2) and

$$
\operatorname{Det}\left(D+m+\mu \gamma_{4}\right)^{*}=\operatorname{Det}\left(D+m-\mu^{*} \gamma_{4}\right) \text {. }
$$

Also in lattice QCD, the fermionic kernel $D_{F}$ corresponding to $D+m$ generally satisfies $D_{F}^{\dagger}=\gamma_{5} D_{F} \gamma_{5}$ [6], and therefore one finds

$$
\left[\bar{\psi}\left(D_{F}+\mu \gamma_{4}\right) \psi\right]^{*}=\bar{\psi}\left(D_{F}-\mu^{*} \gamma_{4}\right) \psi,
$$

which leads to Equation (2) and

$$
\operatorname{Det}\left(D_{F}+\mu \gamma_{4}\right)^{*}=\operatorname{Det}\left(D_{F}-\mu^{*} \gamma_{4}\right) \text {. }
$$

Then, as an exceptional case, the QCD action with the pure imaginary chemical potential $\mu \in i \mathbf{R}$ is manifestly real, and hence its lattice calculation is free from the sign problem. However, the QCD action is generally complex at finite density.

\subsection{Definition and Setup of Matter-Antimatter Coexistence Method}

Now, we show the definition and setup of our approach, the "matter-antimatter coexistence method" [5]. In this method, we consider matter and anti-matter systems on two parallel $\mathbf{R}^{4}$-sheets in five-dimensional Euclidean space-time. For the matter system $M$ with a chemical potential $\mu \in \mathbf{C}$ on a $\mathbf{R}^{4}$-sheet, we also prepare the anti-matter system $\bar{M}$ with $-\mu^{*}$ on the other $\mathrm{R}^{4}$-sheet shifted in the fifth direction, as shown in Figure 1.

We put an ordinary fermion field $\psi(x)$ with the mass $m$ and the gauge variable $U_{v}(x) \equiv \mathrm{e}^{\operatorname{iag} A_{v}(x)}$ at $x \in \mathbf{R}^{4}$ on the matter system $M$, and put the other 


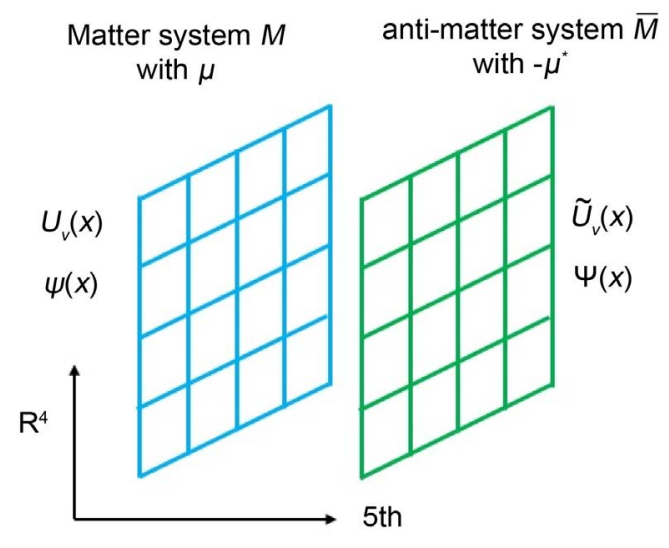

Figure 1. The matter-antimatter coexistence system in five-dimensional Euclidean space-time. We put the matter system $M$ with $\mu, U_{v}(x)$ and $\psi(x)$ on a $\mathbf{R}^{4}$-sheet, and the anti-matter system $\bar{M}$ with $-\mu^{*}, \quad \tilde{U}_{v}(x)=U_{v}(x+\hat{5})$ and $\Psi(x)=\psi(x+\hat{5})$ on the other $\mathbf{R}^{4}$-sheet shifted in the fifth direction.

fermion field $\Psi(x) \equiv \psi(x+\hat{5})$ with the same mass $m$ and the gauge variable $\tilde{U}_{v}(x) \equiv \mathrm{e}^{i a g \tilde{A}_{v}(x)} \equiv U_{v}(x+\hat{5})$ on the anti-matter system $\bar{M}$. Here, $\hat{5}$ denotes the fifth direction vector with an arbitrary length $a_{5}$, which is independent of four-dimensional lattice spacing $a$.

Between the gauge variables $U_{v}(x)$ in $M$ and $\tilde{U}_{v}(x)$ in $\bar{M}$, we introduce a correlation term such as

$$
S_{\lambda} \equiv \sum_{x, \nu} 2 \lambda\left\{N_{c}-\operatorname{Re} \operatorname{tr}\left[U_{v}(x) \tilde{U}_{v}^{\dagger}(x)\right]\right\}
$$

with a real parameter $\lambda \quad(\geq 0)$ in the lattice formalism. This correlation acts on $U_{v}(x)$ and $\tilde{U}_{v}(x)$ at the same four-dimensional coordinate $x \in \mathbf{R}^{4}$, and connects two different situations: $\tilde{U}_{v}(x)=U_{v}(x)$ in $\lambda \rightarrow \infty$ and two separated QCD systems in $\lambda \rightarrow 0$.

In fact, the total lattice action in this method is expressed as

$$
\begin{aligned}
S= & S_{G}[U]+\sum_{x} \bar{\psi}\left(D_{F}[U]+\mu \gamma_{4}\right) \psi+S_{G}[\tilde{U}] \\
& +\sum_{x} \bar{\Psi}\left(D_{F}[\tilde{U}]-\mu^{*} \gamma_{4}\right) \Psi+\sum_{x, v} 2 \lambda\left\{N_{c}-\operatorname{Re} \operatorname{tr}\left[U_{v}(x) \tilde{U}_{v}^{\dagger}(x)\right]\right\}
\end{aligned}
$$

with the gauge action $S_{G}[U] \in \mathbf{R}$ and the fermionic kernel $D_{F}[U]$ in lattice QCD. After integrating out the fermion fields $\psi$ and $\Psi$, the generating functional of this theory reads

$$
\begin{aligned}
Z= & \int D U \mathrm{e}^{-S_{G}[U]} \operatorname{Det}\left(D_{F}[U]+\mu \gamma_{4}\right) \int D \tilde{U} \mathrm{e}^{-S_{G}[\tilde{U}]} \operatorname{Det}\left(D_{F}[\tilde{U}]-\mu^{*} \gamma_{4}\right) \\
& \times \mathrm{e}^{-\sum_{x, \nu} 2 \lambda\left\{N_{c}-\operatorname{Retr}\left[U_{v}(x) \tilde{U}_{v}^{+}(x)\right]\right\}} \\
= & \int D U \int D \tilde{U} \mathrm{e}^{-\left(S_{G}[U]+S_{G}[\tilde{U}]\right)} \operatorname{Det}\left\{\left(D_{F}[U]+\mu \gamma_{4}\right)\left(D_{F}[\tilde{U}]-\mu^{*} \gamma_{4}\right)\right\} \\
& \times \mathrm{e}^{-\sum_{x, \nu} 2 \lambda\left\{N_{c}-\operatorname{Retr}\left[U_{v}(x) \tilde{U}_{v}^{+}(x)\right]\right\}} .
\end{aligned}
$$


Here, the total fermionic determinant

$$
\Delta[U, \tilde{U}] \equiv \operatorname{Det}\left\{\left(D_{F}[U]+\mu \gamma_{4}\right)\left(D_{F}[\tilde{U}]-\mu^{*} \gamma_{4}\right)\right\}
$$

is generally complex, and all other factors are real and non-negative in $Z$.

\subsection{Four-Dimensional Continuum Limit}

Near the four-dimensional continuum limit of $a \simeq 0$, this additional term becomes

$$
S_{\lambda} \simeq \sum_{x} \frac{1}{2} \lambda a^{2}\left\{A_{v}^{a}(x)-\tilde{A}_{v}^{a}(x)\right\}^{2} \simeq \int \mathrm{d}^{4} x \frac{1}{2} \lambda_{\text {phys }}\left\{A_{\nu}^{a}(x)-\tilde{A}_{\nu}^{a}(x)\right\}^{2}
$$

with $\lambda_{\text {phys }} \equiv \lambda a^{-2}$, and the generating functional goes to

$Z_{\text {cont }}=\int D A \int D \tilde{A} \mathrm{e}^{-\left(S_{G}[A]+S_{G}[\tilde{A}]\right)} \operatorname{Det}\left\{\left(D+m+\mu \gamma_{4}\right)\left(\tilde{D}+m-\mu^{*} \gamma_{4}\right)\right\} \mathrm{e}^{-\int \mathrm{d}^{4} x \frac{1}{2} \lambda_{\text {phys }}\left\{A_{r}^{a}(x)-\tilde{A}_{v}^{a}(x)\right\}^{2}}$

with the continuum gauge action $S_{G}[A] \in \mathbf{R}$ and $\tilde{D}^{v} \equiv \partial^{v}+i g \tilde{A}^{v}$.

\subsection{Lattice Calculation Procedure}

Next, we show the actual procedure of this method for the lattice QCD calculation at finite density. In the practical lattice calculation with the Monte Carlo method, the fermionic determinant in $Z$ is factorized into its amplitude and phase factor as

$$
\begin{aligned}
Z= & \int D U \int D \tilde{U} \mathrm{e}^{-\left(s_{G}[U]+S_{G}[\tilde{U}]\right)}\left|\operatorname{Det}\left\{\left(D_{F}[U]+\mu \gamma_{4}\right)\left(D_{F}[\tilde{U}]-\mu^{*} \gamma_{4}\right)\right\}\right| \\
& \times \mathrm{e}^{-\sum_{x, V} 2 \lambda\left\{N_{c}-\operatorname{Ret} \operatorname{tr}\left[U_{v}(x) \tilde{U}_{v}^{\dagger}(x)\right]\right\}} O_{\text {phase }}[U, \tilde{U}],
\end{aligned}
$$

and the phase factor of the total fermionic determinant

$$
O_{\text {phase }}[U, \tilde{U}] \equiv \mathrm{e}^{\operatorname{iarg}\left[\operatorname{Det}\left\{\left(D_{F}[U]+\mu \gamma_{4}\right)\left(D_{F}[\tilde{U}]-\mu^{*} \gamma_{4}\right)\right\}\right]}
$$

is treated as an "operator" instead of a probability factor in a similar way to the phase quenched reweighting method [3], while all other real non-negative factors in $Z$ can be treated as the probability density.

The additional term $S_{\lambda}$ connects the following two different situations as the two limits of the parameter $\lambda$ :

1) In one limit of $\lambda \rightarrow \infty$, a strong constraint $\tilde{U}_{v}(x)=U_{v}(x)$ is realized, and the phase factors of two fermionic determinants $\operatorname{Det}\left(D_{F}[U]+\mu \gamma_{4}\right)$ and $\operatorname{Det}\left(D_{F}[\tilde{U}]-\mu^{*} \gamma_{4}\right)$ are completely cancelled, owing to Equation (6). Therefore, the total fermionic determinant is real and non-negative,

$$
\operatorname{Det}\left\{\left(D_{F}[U]+\mu \gamma_{4}\right)\left(D_{F}[\tilde{U}=U]-\mu^{*} \gamma_{4}\right)\right\} \geq 0 \text {, }
$$

that is, $O_{\text {phase }}[U, \tilde{U}]=1$, and the numerical calculation becomes possible without the sign problem. Note however that this system resembles QCD with an isospin chemical potential [7], which is different from finite density QCD.

2) In another limit of $\lambda \rightarrow 0$, this system goes to "two separated ordinary QCD systems" with the chemical potential of $\mu$ and $-\mu^{*}$, although the 
cancellation of the phase factors cannot be expected between the two fermionic determinants $\operatorname{Det}\left(D_{F}[U]+\mu \gamma_{4}\right)$ and $\operatorname{Det}\left(D_{F}[\tilde{U}]-\mu^{*} \gamma_{4}\right)$ for significantly different $U_{v}(x)$ and $\tilde{U}_{v}(x)$, which are independently generated in the Monte Carlo simulation.

In fact, in other words, this approach links QCD with a chemical potential and QCD with an isospin-chemical potential.

For a given four-dimensional finite-volume lattice, if one takes an enough large value of $\lambda, \tilde{U}_{v}(x) \simeq U_{v}(x)$ is realized, and approximate phase cancellation occurs between the two fermionic determinants $\operatorname{Det}\left(D_{F}[U]+\mu \gamma_{4}\right)$ and $\operatorname{Det}\left(D_{F}[\tilde{U}]-\mu^{*} \gamma_{4}\right)$ in $M$ and $\bar{M}$. Then, we expect a modest behavior of the phase factor $O_{\text {phase }}[U, \tilde{U}]$ in Equation (14), which leads to feasibility of the numerical lattice calculation with suppression of the sign problem.

Once the lattice gauge configurations of the coexistence system are obtained with the most importance sampling in the Monte Carlo simulation, matter-side quantities can be evaluated through their measurement only for the matter part $M$ with $\mu$.

By performing the lattice calculations with gradually decreasing $\lambda$ and their extrapolation to $\lambda=0$, we expect to estimate the physical quantities in finite density QCD with the chemical potential $\mu$. (This procedure may resemble the chiral extrapolation, where the current quark mass $m$ is gradually reduced and the lattice data is extrapolated to $m=0$.)

Here, we summarize the lattice QCD procedure of the matter-antimatter coexistence method for finite density QCD:

1) For the matter system $M$ with $\mu$ on a finite-volume lattice, prepare also the anti-matter system $\bar{M}$ with $-\mu^{*}$ on a fifth-dimensional parallel lattice.

2) For the coexistence system $M+\bar{M}$, introduce the correlation $S_{\lambda}$ between $M$ and $\bar{M}$.

3) For the finite four-dimensional volume $V$, take an enough large value of $\lambda$ so as to realize approximate phase cancellation in the total fermionic determinant $\Delta[U, \tilde{U}]$, and generate the gauge configurations using the Monte Carlo calculation, with treating the phase factor $O_{\text {phase }}[U, \tilde{U}]$ as an operator.

4) For the obtained gauge configurations of the coexistence system, perform the lattice QCD measurement only for the matter part $M$ with $\mu$.

5) Reduce the value of the correlation parameter $\lambda$ gradually, and extrapolate the lattice data to $\lambda=0$.

As a caution, even with this method, it is rather difficult to deal with the large volume case, because the phase factor $O_{\text {phase }}[U, \tilde{U}]$ largely changes depending on the gauge configuration in the large volume, when $\lambda$ is reduced. For the finite volume, however, this method is expected to suppress the sign problem and to enlarge the calculable region at finite density and temperature.

\subsection{More Sophisticated Correlation between Matter and Antimatter Systems}

In this method, we have introduced an additional correlation between the matter 
$M$ and the anti-matter $\bar{M}$. Although the system goes to ordinary QCD in the limit of $\lambda \rightarrow 0$, it is desired to reduce some effect from the additional correlation at finite values of $\lambda$. On this point, we here consider a possible improvement in this framework.

So far, we have demonstrated this method by taking the simplest correlation of $S_{\lambda}$ in Equation (7). In this method, however, there is some variety on the choice of the correlation between $U_{v}(x)$ in $M$ and $\tilde{U}_{v}(x)$ in $\bar{M}$. In fact, the validity of the data extrapolation can be checked by various extrapolations with different type of the additional correlation.

In particular, it is interesting to consider more sophisticated correlation such as

$$
\bar{S}_{\xi} \equiv \sum_{x} 8 \xi\left(\sum_{v}\left\{N_{c}-\operatorname{Retr}\left[U_{v}(x) \tilde{U}_{v}^{\dagger}(x)\right]\right\}\right)^{3} \simeq \int \mathrm{d}^{4} x \frac{1}{8} a^{2} \xi\left[\left\{A_{v}^{a}(x)-\tilde{A}_{v}^{a}(x)\right\}^{2}\right]^{3}
$$

with a dimensionless non-negative real parameter $\xi$. At the classical level, this correlation is an irrelevant interaction and it gives vanishing contributions in the continuum limit $a \rightarrow 0$, like the Wilson term $-\frac{1}{2} \operatorname{ar} \bar{\psi} D^{2} \psi$ [6]. By the use of this irrelevant-type correlation, the effect from the additional term is expected to be reduced in the actual lattice calculation.

\section{Summary and Conclusion}

We have proposed the "matter-antimatter coexistence method" toward the lattice calculation of finite density QCD. In this method, we have prepared matter $M$ with $\mu$ and anti-matter $\bar{M}$ with $-\mu^{*}$ on two parallel $\mathbf{R}^{4}$-sheets in five-dimensional Euclidean space-time, and have introduced a correlation term $S_{\lambda} \equiv \sum_{x, v} 2 \lambda\left\{N_{c}-\operatorname{Retr}\left[U_{v}(x) \tilde{U}_{v}^{\dagger}(x)\right]\right\} \simeq \sum_{x} \frac{1}{2} \lambda a^{2}\left\{A_{v}^{a}(x)-\tilde{A}_{v}^{a}(x)\right\}^{2}$ between the gauge variables $U_{v}=\mathrm{e}^{i a g A_{v}}$ in $M$ and $\tilde{U}_{v}=\mathrm{e}^{i a g \tilde{A}_{v}}$ in $\bar{M}$. In one limit of $\lambda \rightarrow \infty$, owing to $\tilde{U}_{v}(x)=U_{v}(x)$, the total fermionic determinant is real and non-negative, and the sign problem is absent. In another limit of $\lambda \rightarrow 0$, this system goes to two separated ordinary QCD systems with the chemical potential of $\mu$ and $-\mu^{*}$.

For a given finite-volume lattice, if one takes an enough large value of $\lambda$, $\tilde{U}_{v}(x) \simeq U_{v}(x)$ is realized and phase cancellation approximately occurs between two fermionic determinants in $M$ and $\bar{M}$, which is expected to suppress the sign problem and to make the lattice calculation possible. For the obtained gauge configurations of the coexistence system, matter-side quantities can be evaluated by their measurement only for the matter part $M$. By gradually reducing $\lambda$ and the extrapolation to $\lambda=0$, it is expected to obtain estimation of the physical quantities in finite density QCD with $\mu$.

The next step is to perform the actual lattice QCD calculation at finite density using this method. It would be useful to combine this method with the other known ways such as the hopping parameter expansion [8], the complex 
Langevin method [2] and the reweighting technique [3]. For example, if the hopping parameter expansion is utilized, huge calculations of the fermionic determinant can be avoided, and a low-cost analysis with the quenched gauge configuration becomes possible, since the additional term $S_{\lambda}$ only includes gauge variables. In addition to the actual lattice calculation, the effect from the additional term is to be investigated carefully.

Efficiency of this method would strongly depend on the system parameters, such as the space-time volume $V$, the quark mass $m$, the temperature $T$ and the chemical potential $\mu$. For instance, near the chiral limit $m=0$ in large $V$, the fermionic determinant tends to possess quasi-zero-eigenvalues, which permits a drastic change of the phase in the fermionic determinant, although the zero fermionic-determinant case would give no significant contribution in the QCD generating functional. In any case, this method is expected to enlarge calculable area of the QCD phase diagram on $(T, \mu, m, V)$.

\section{Acknowledgements}

The author is supported in part by the Grants-in-Aid for Scientific Research (Grant No. 15K05076) from Japan Society for the Promotion of Science.

\section{References}

[1] Creutz, M. (1980) Physical Review D, 21, 2308-2315. https://doi.org/10.1103/PhysRevD.21.2308

[2] Parisi, G. (1983) Physics Letters B, 131, 393-395. https://doi.org/10.1016/0370-2693(83)90525-7

[3] Fodor, Z. and Katz, S.D. (2002) Physics Letters B, 534, 87-92. https://doi.org/10.1016/S0370-2693(02)01583-6

[4] Hasenfratz, P. and Karsch, F. (1984) Physics Report, 103, 219-223. https://doi.org/10.1016/0370-1573(84)90083-8

[5] Suganuma, H. (2017) Matter-Antimatter Coexistence Method for Finite Density QCD. Proceedings of the International Workshop on Excited QCD 2017, Sintra, 7-13 May 2017, arXiv: 1707.05996 [hep-lat], to appear in Acta Physica Polonica B Proceedings Supplement.

[6] Rothe, H.J. (2012) Lattice Gauge Theories. World Scientific, Singapore. https://doi.org/10.1142/8229

[7] Son, D.T. and Stephanov, M.A. (2001) Physical Review Letters, 86, 592-595. https://doi.org/10.1103/PhysRevLett.86.592

[8] Aarts, G., Seiler, E., Sexty, D. and Stamatescu, I.O. (2014) Physical Review D, 90, Article ID: 114505. https://doi.org/10.1103/PhysRevD.90.114505 